\title{
Fullerenes in Circumstellar and Interstellar Environments
}

\author{
Jan Cami \\ Department of Physics \& Astronomy, The University of Western Ontario, \\ London ON N6A 3K7, Canada \\ email: jcami@uwo.ca
}

\begin{abstract}
In recent years, the fullerene species $\mathrm{C}_{60}$ (and to a lesser extent also $\mathrm{C}_{70}$ ) has been reported in the mid-IR spectra of various astronomical objects. Cosmic fullerenes form in the circumstellar material of evolved stars, and survive in the interstellar medium (ISM). It is not entirely clear how they form or what their excitation mechanism is.
\end{abstract}

Keywords. Astrochemistry, (stars:) circumstellar matter, ISM: molecules, infrared: ISM

\section{Fullerenes in astrophysical environments}

Fullerenes (such as the buckminsterfullerene $\mathrm{C}_{60}$ ) are large carbonaceous molecules in the shape of a hollow sphere of ellipsoid. They are very stable, and thus it was suggested early on that they could also form in space and be abundant and widespread in the Universe (Kroto et al. 1985). Astronomical searches for the electronic bands of neutral $\mathrm{C}_{60}$ were unsuccessful though (for an overview, see Herbig 2000); and the detection of two diffuse interstellar bands near the predicted wavelengths of $\mathrm{C}_{60}^{+}$awaits confirmation from a gas-phase laboratory spectrum (Foing \& Ehrenfreund 1994). $\mathrm{C}_{60}$ also has $4 \mathrm{IR}$ active vibrational modes at 7.0, 8.5, 17.4 and $18.9 \mu \mathrm{m}$. Dedicated searches for these bands did not result in a detection either (Clayton et al. 1995; Moutou et al. 1999).

Recently, we reported the detection of the IR active modes of $\mathrm{C}_{60}$ and $\mathrm{C}_{70}$ in the Spitzer-IRS spectrum of the young, low-excitation planetary nebula (PN) Tc 1 (Cami et al. 2010). Since then, fullerenes have been found in many more PNe (García-Hernández et al. 2010, 2011a), a proto-PN (Zhang \& Kwok 2011), a few R Cor Bor stars (GarcíaHernández et al. 2011b; Clayton et al. 2011) and even in O-rich binary post-AGB stars (Gielen et al. 2011). In addition, fullerenes have turned up in interstellar environments (Sellgren et al. 2010; Rubin et al. 2011; Boersma et al. 2012) and in young stellar objects (Roberts et al. 2012) as well. From these detections it is clear that fullerenes are formed in the circumstellar environments of evolved stars. They then either survive in the ISM (possibly incorporated into dust grains), or form there when conditions are right.

\section{The fullerene excitation mechanism}

To explain the IR emission of cosmic fullerenes, two mechanisms have been considered that offer quite different predictions about the relative band strengths (for a detailed comparison, see Bernard-Salas et al. 2012). Thermal $\mathrm{C}_{60}$ emission models show large variations in the relative strength of all bands as a function of temperature; for $T \leqslant 300 \mathrm{~K}$, the 7.0 and $8.5 \mu \mathrm{m}$ bands are very weak compared to the 17.4 and $18.9 \mu \mathrm{m}$ bands. For fluorescence on the other hand, the band strengths only depend on the average 
absorbed photon energy; in that case, the $17.4 / 18.9 \mu \mathrm{m}$ band ratio is roughly constant (for reasonable photon energies) while the 7.0 and $8.5 \mu \mathrm{m}$ bands should be fairly strong.

Observationally, the 7.0 and $8.5 \mu \mathrm{m}$ bands are often very weak or even undetectable, while there are considerable variations in the $17.4 / 18.9 \mu \mathrm{m}$ band ratio; this is more easily explained by thermal models than by fluorescence models. However, these variations could also be the consequence of contamination by PAH emission. In the three known uncontaminated fullerene-rich $\mathrm{PNe}$ on the other hand, the $7.0 \mu \mathrm{m}$ band is far too strong to be explained by even fluorescence from $\mathrm{C}_{60}$ alone. As pointed out to the careful reader by Bernard-Salas et al. (2012), the $7.0 \mu \mathrm{m}$ emission in those sources includes a significant contribution from $\mathrm{C}_{70}$, provided at least that the emission is due to fluorescence. For one object (Tc 1), fluorescence is further supported by the observation that the $\mathrm{C}_{60}$ emission peaks at large distances $(\sim 8000 \mathrm{AU})$ from the central star. If fluorescence is also the excitation mechanism for the other astronomical sources where fullerenes have been detected, then the weak 7.0 and $8.5 \mu \mathrm{m}$ bands indicate that the fullerene emission is not due to isolated, free $\mathrm{C}_{60}$ molecules in the gas-phase; there might be contributions from other species as well and/or the emission may be due to fullerene clusters or nanocrystals.

\section{The formation of cosmic fullerenes}

Several routes have been proposed to explain the formation of fullerenes in astrophysical environments. Densities in circumstellar and interstellar environments are too low for bottom-up fullerene formation on reasonable timescales (Micelotta et al. 2012). Fullerenes could form from the processing of PAHs (Berné \& Tielens 2012), but this requires fine-tuned initial conditions. A promising route starts from arophatics - large clusters of aromatic rings with aliphatic and olefinic bridges that originate from a:C-H grains (Micelotta et al. 2012). UV irradiation first dehydrogenates and aromatizes such structures; subsequent $\mathrm{C}_{2}$ ejection then shrinks down the resulting cages to $\mathrm{C}_{60}$. Further shrinking is inhibited by a high energy barrier. The spectral imprint of the parent a:C-H grains in the IR spectra of fullerene-rich PNe offers some observational support for this mechanism (Bernard-Salas et al. 2012).

\section{References}

Bernard-Salas, J., Cami, J., Peeters, E., et al. 2012, ApJ 757, 41

Berné, O. \& Tielens, A. G. G. M., 2012, PNAS 109, 401

Boersma, C., Rubin, R. H., \& Allamandola, L. J. 2012, ApJ 753, 168

Cami, J., Bernard-Salas, J., Peeters, E., \& Malek, S. E. 2010, Science 329, 1180

Clayton, G. C., De Marco, O., Whitney, B. A., et al. 2011, AJ 142, 54

Clayton, G. C., Kelly, D. M., Lacy, J. H., et al. 1995, AJ 109, 2096

Foing, B. H. \& Ehrenfreund, P. 1994, Nature 369, 296

García-Hernández, D. A., Iglesias-Groth, S., Acosta-Pulido, J. A., et al. 2011a, ApJ 737, L30

García-Hernández, D. A., Kameswara Rao, N., \& Lambert, D. L. 2011b, ApJ 729, 126

García-Hernández, D. A., Manchado, A., García-Lario, P., et al. 2010, ApJ 724, L39

Gielen, C., Cami, J., Bouwman, J., Peeters, E., \& Min, M. 2011, A\&A 536, A54

Herbig, G. H. 2000, ApJ 542, 334

Kroto, H. W., Heath, J. R., Obrien, S. C., Curl, R. F., \& Smalley, R. E. 1985, Nature 318, 162

Micelotta, E., Jones, A. P., Cami, J., et al. 2012, ApJ 761, 35

Moutou, C., Sellgren, K., Verstraete, L., \& Léger, A. 1999, A\&A 347, 949

Roberts, K. R. G., Smith, K. T., \& Sarre, P. J. 2012, MNRAS 421, 3277

Rubin, R. H., Simpson, J. P., O'Dell, C. R., et al. 2011, MNRAS 410, 1320

Sellgren, K., Werner, M. W., Ingalls, J. G., et al. 2010, ApJ 722, L54

Zhang, Y. \& Kwok, S. 2011, ApJ 730, 126 